\title{
Stochastic field-induced nonlocal resonances in four-wave mixing
}

\author{
M. Belsley \\ Departamento de Física, Universidade do Minho, 4709 Braga, Portugal \\ M. Kaczmarek \\ Department of Physics and Astronomy, University of Southampton, Southampton SO17 1BJ, United Kingdom \\ P. Ewart \\ Oxford Institute for Laser Science, Clarendon Laboratory, University of Oxford, Oxford OX1 3PU, United Kingdom
}

(Received 17 August 1999; revised manuscript received 27 April 2001; published 14 November 2001)

\begin{abstract}
We explore theoretically the signal generated by a fluctuating radiation field via a four-wave mixing process in an atomic vapor undergoing Markovian dephasing collisions. We consider specifically a broadband laser field well detuned from the atomic resonance and calculate the signal generated using counterpropagating pump fields as a function of the time delay of the incident probe field. We assume that the pulse duration is long compared to the correlation time of the incident fields. By considering the various time-ordered pathways and treating the incident-field amplitudes as random Gaussian variables, we find that the signal generated is dominated by two specific pathways that depend only on the incident-field bandwidth and the decay rate of the ground to excited state coherence. An interesting aspect of the far-impact-detuning case is that the signal is dominated by resonances involving spatially separated atoms. Our formalism provides a basis for describing the effects of mode correlations on four-wave-mixing signals generated by stochastic fields.
\end{abstract}

DOI: 10.1103/PhysRevA.64.063806

PACS number(s): 42.65.-k, 42.50.- p

\section{INTRODUCTION}

Coherent signals induced by fundamentally incoherent phenomena through a four-wave-mixing process are among the most intriguing and subtle effects in nonlinear spectroscopy. Bloembergen and co-workers first introduced such effects by considering pressure-induced extra resonances in four-wave mixing (PIER4) [1] as arising from the partial cancellation, by collisions, of the destructive interference between alternative time-ordered pathways contributing to the third-order nonlinear susceptibility $\chi^{(3)}$. Alternative descriptions of such "destruction of destructive interferences" have been given in terms of energy conservation, by analogies with collisional redistribution, and in terms of dressed atomic states [2]. Early on Agarwal and Kunasz [3] and Prior and co-workers $[4,5]$ noted that phase fluctuations in a stochastic radiation field could produce resonances in the nonlinear atomic susceptibility in a similar manner to the collisioninduced resonances. The influence of these field-fluctuationinduced resonances can be experimentally investigated by subjecting one of the incident radiation fields to a variable time delay ranging up to several correlation times of the incident field [6].

The general problem of adequately describing nonlinear optical processes produced by the interaction of time-delayed fluctuating laser fields with an atomic system connected to a thermal reservoir has stimulated a large body of recent work $[6-11]$ From a theoretical point of view, the problem is particularly interesting due to the multiple (and often similar) time scales associated with the evolution of the incident fields, the isolated atomic system, and the Rabi frequencies of the driven atomic system. In addition, multiple interactions with the incident field make the atomic system sensitive to the higher-order statistics of the light field. Indeed, even in the case in which the fluctuations of the incident field are Markovian, the atomic system may retain some memory of the first field interaction when a second interaction occurs, making the entire process non-Markovian [10,11].

Initial work $[7,12]$ was motivated by the possibility of using broadband stochastic fields to achieve femtosecond scale time resolution in nonlinear spectroscopy experiments. The original theoretical treatments assumed a $\delta$-function correlation for the incident fields and proceeded to solve the Liouville equations for the density matrix operator perturbatively to obtain the material response of a two-level atom (or molecule) to a (white-light) stochastic field. Related work by Agarwal and co-workers $[3,8]$ and by Kofman, Levine, and Prior [5] considered the possibility of achieving stochasticfluctuation-induced resonances similar to the PIER resonances predicted and observed by Prior et al. [1] A rather complete exposition of the general problem from the viewpoint of a molecular spectroscopist was recently given by Albrecht and co-workers [13,14]. In all the above cited cases the authors limited themselves to considering the actions of fields tuned close to an atomic or molecular resonance. They were primarily concerned with developing techniques using stochastic fields to obtain spectroscopic information regarding the active atoms or molecules. Working close to resonance, the dominant terms are due to resonances involving single-atom transitions, which generally allows one to express the signal in terms of an effective nonlinear susceptibility [3].

Very recently Bratfalean and Ewart [15] considered the case of far-nonresonant interactions leading to the generation of a four-wave-mixing signal in the phase-conjugate geometry. In particular, they sought to describe a situation in which the frequency spectrum of the generated signal field was determined solely by the statistics of the incident field, 


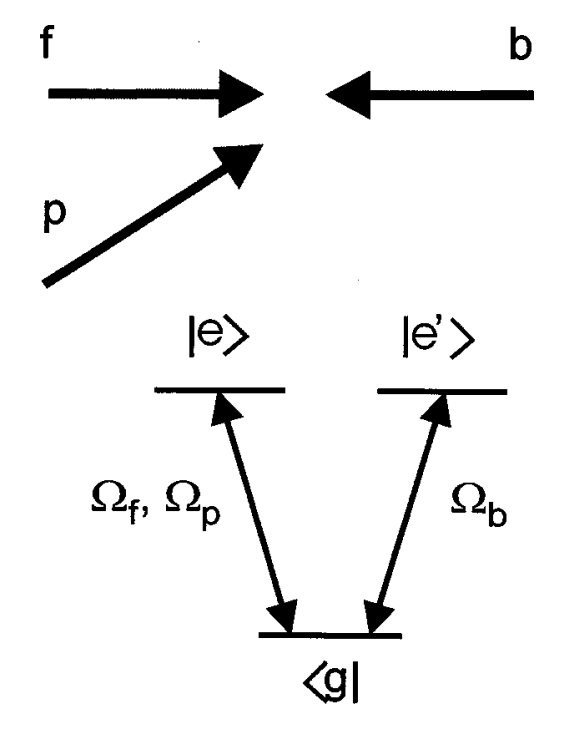

FIG. 1. The four-wave-mixing phase-conjugate geometry. The incident fields consist of a forward-propagating pump beam with Rabi frequency $\Omega_{f}$, a probe beam with Rabi frequency $\Omega_{p}$, and a backward-going pump beam with Rabi frequency $\Omega_{b}$. The atomic medium is taken to be a set of two-level, three-state atoms with the forward pump and probe beams inducing transitions between the ground state $|g\rangle$ and the excited state $|e\rangle$. The backward pump interacts with the other degenerate excited state $\left|e^{\prime}\right\rangle$.

for example, by using field detunings that are sufficiently large to ensure that the active atoms interact equally with the entire spectrum. Unfortunately, this involves an assumption that is, in general, unjustified, namely, that the atomic response can be considered separately from the correlations present in the stochastic incident field. Strictly speaking, this can be realized only in systems possessing a vanishingly small atomic or molecular memory time. As we indicate below, in the limit of a thin medium, the four-wave-mixing signal originates from a two-point correlation product between atomic coherences at different spatial locations. Incoherent effects such as collisional dephasing and spontaneous emission act independently at each spatial location and can therefore alter the correlations that exist between the atomic coherences induced by the incident fields at separate locations. Essentially the incoherent atomic decay mechanisms determine the effective time scale over which an atom can "remember" a past interaction with an incident field. The motivation of our work is to explore the interplay between the incident-field statistics and the incoherent processes that influence the atomic or molecular memory by examining in detail the case of far-off-resonance detunings. We consider explicitly the case for which all three incident fields are correlated and show that in the "far-impact region" (defined below) the signal is dominated by terms containing "nonlocal" resonances between two different atoms. As we discuss in detail below, such nonlocal resonances cannot be described by an effective nonlinear susceptibility and the usual Maxwell-Bloch treatment of four-wave mixing becomes inadequate in this limit. To our knowledge, such nonlocal resonances have not been described before in the context of timedelayed four-wave-mixing experiments in atomic vapors.

In the following section we briefly present the context of

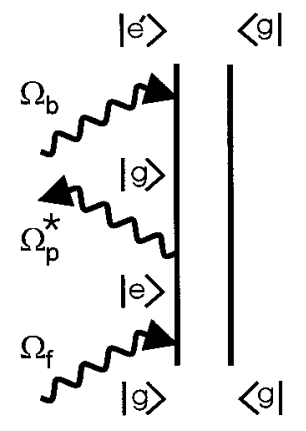

(a) Pathway I

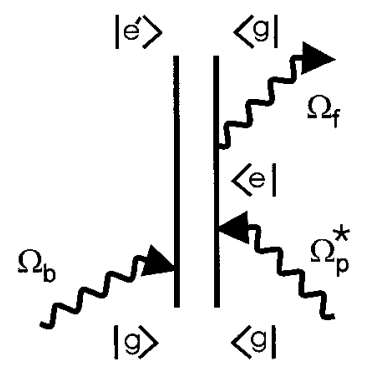

(c) Pathway III

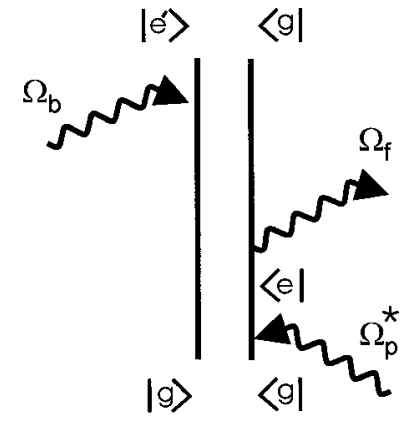

(b) Pathway II

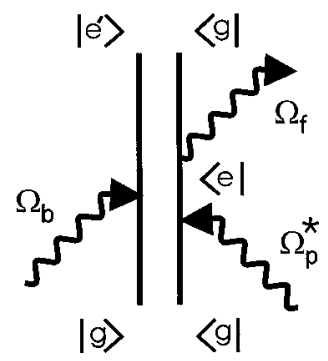

(d) Pathway IV
FIG. 2. The double-sided Feynman diagrams showing the four possible time-ordered pathways for creating a third-order coherence $\rho_{e^{\prime} g}^{f, p^{*}, b}$.

the problem and write down the relevant equations. We then go on to present a detailed calculation of the phase-conjugate four-wave-mixing signal generated by a stochastic field detuned by many atomic linewidths from an atomic resonance. We show that the signal in this case is dominated by a small subset of the available time-ordered pathways. After taking into account the averages over the atomic motion, we conclude by analyzing the dependence of the generated phaseconjugate signal on the collisional dephasing rate and the bandwidths of the incident radiation fields.

\section{FORMULATION OF THE PROBLEM}

To explore these concepts within a manageable theoretical framework, we consider the case of three broadband laser fields interacting with a two-level, three-state atomic system (V system) in the phase-conjugate geometry (see Fig. 1). The copolarized forward pump and probe beams, with electric field amplitudes $E_{f}$ and $E_{p}$, couple the ground state $|g\rangle$ with the excited state $|e\rangle$ while a perpendicularly polarized backward (i.e., counterpropagating) pump beam, with electric field amplitude $E_{b}$, couples $|g\rangle$ to the degenerate excited state $\left|e^{\prime}\right\rangle$. We monitor the phase-conjugate signal, polarized parallel to the backward-going pump beam, as a function of the time delay $\tau$ between the probe field and the two pump fields. Adopting a semiclassical approach, all incident fields are taken to be classical plane waves while the angle between the incident forward-going pump and probe waves is taken to be vanishingly small. 
Within the slowly varying envelope approximation the phase-matching condition implies that the signal field will propagate antiparallel to the incident probe wave, i.e., with a wave vector $\mathbf{k}_{s}=-\mathbf{k}_{p}$ and with a polarization parallel to that of the backward-going pump beam. As mentioned in the Introduction, the interplay between collisional dephasing and field fluctuations is best seen in what we call the far impact region of the atomic absorption spectrum. This region is defined by detunings from line center, $\Delta$, large enough to ensure that all frequencies contained in the incident field interact equally strongly with the atomic resonance but small enough to retain the Markovian aspects of the usual impact approximation for collisional dephasing. Thus, for incident fields with a bandwidth $B$ and an atomic line with homogeneous and inhomogeneous widths $\gamma_{h}$ and $\gamma_{\mathrm{inh}}$, respectively, the far-impact region is defined by $\left(B, \gamma_{h}, \gamma_{i n h} \ll|\Delta| \ll \tau_{c}^{-1}\right)$, where $\tau_{c}$ is the duration of a typical dephasing collision. For a typical atomic vapor, this condition will result in a detuning $|\Delta|$ of the order of a few $\mathrm{cm}^{-1}$.

Within this limit the induced atomic polarization that gives rise to the phase-conjugate signal may be calculated perturbatively [7], being proportional to the density matrix element $\rho_{e^{\prime} g}^{f, p^{*}, b}$, where the superscripts denote single interactions with the forward and backward pumps $(f$ and $b)$ and the probe field $p$. To lowest order the number of distinct time-ordered pathways that contribute to the signal is limited by phase-matching considerations to the four paths displayed in the double Feynman graphs of Fig. 2.

The detected signal $S$ is proportional to the modulus squared of the phase-conjugate field generated by the sample. It has been shown by several authors $[16,8]$ that the signal (integrated over the pulse) is proportional to a twopoint spatial correlation function of the form

$$
S \propto \int d t d \mathbf{r} d \mathbf{r}^{\prime} \sum_{j, k=\mathrm{I}, \mathrm{II}, \mathrm{III}, \mathrm{IV}}\left\langle\rho_{e^{\prime} g}^{j}(\mathbf{r}, \mathbf{v}, t)\left[\rho_{e^{\prime} g}^{k}\left(\mathbf{r}^{\prime}, \mathbf{v}^{\prime}, t\right)\right]^{*}\right\rangle .
$$

The angular brackets denote averages over both the field fluctuations and the velocities $\mathbf{v}$ and $\mathbf{v}^{\prime}$. By writing the same time $t$ for both third-order atomic coherences in the above expression, we are making the usual approximation as pointed out by Mukamel and Hanamura [16]; the signal cannot, in general, be factored into an amplitude squared as is the case with an approach based on the Maxwell-Bloch equations. In our case, as we show in detail below, it is the stochastic nature of the incident radiation fields that leads to nonfactorable correlations between the active atoms at points $\mathbf{r}$ and $\mathbf{r}^{\prime}$.

\section{CALCULATION OF THE FOUR-WAVE MIXING SIGNAL}

As there are four possible pathways leading to the coherence $\rho_{e^{\prime} g}^{f, p^{*}, b}(\mathbf{r}, \mathbf{v}, t)$, the angular brackets in Eq. (1) contain the sum of 16 different six-dimensional integrals, each one containing a sixth-order correlation function of the incident fields. For example, the six-dimensional integral for the contribution from the time-ordered pathways III and IV takes the form

$$
\begin{aligned}
\left\langle\rho_{e^{\prime} g}^{\mathrm{III}}\left[\rho_{e^{\prime} g}^{\mathrm{IV}}\right]^{*}\right\rangle= & e^{i \mathbf{k}_{s} \cdot\left(\mathbf{r}-\mathbf{r}^{\prime}\right)-i \mathbf{k}_{s} \cdot\left(\mathbf{v}-\mathbf{v}^{\prime}\right) t} \int_{-\infty}^{t} d t_{3} e^{-i\left(\omega_{f}-\mathbf{k}_{f} \cdot \mathbf{v}\right) t_{3}} e^{-\left(i \omega_{0}+\gamma_{e g}\right)\left(t-t_{3}\right)} \\
& \times \int_{-\infty}^{t_{3}} d t_{2} e^{i\left(\omega_{p}-\mathbf{k}_{p} \cdot \mathbf{v}\right) t_{2}} e^{-\gamma_{e^{\prime}}\left(t_{3}-t_{2}\right)} \int_{-\infty}^{t_{2}} d t_{1} e^{-i\left(\omega_{b}-\mathbf{k}_{b} \cdot \mathbf{v}\right) t_{1}} e^{-\left(i \omega_{0}+\gamma_{e g}\right)\left(t_{2}-t_{1}\right)} \\
& \times \int_{-\infty}^{t} d s_{3} e^{i\left(\omega_{f}-\mathbf{k}_{f} \cdot \mathbf{v}^{\prime}\right) s_{3}} e^{\left(i \omega_{0}-\gamma_{e g}\right)\left(t-s_{3}\right)} \int_{-\infty}^{s_{3}} d s_{2} e^{i\left(\omega_{b}-\mathbf{k}_{b} \cdot \mathbf{v}^{\prime}\right) s_{2}} e^{-\gamma_{e^{\prime} e}\left(s_{3}-s_{2}\right)} \\
& \times \int_{-\infty}^{s_{2}} d s_{1} e^{-i\left(\omega_{p}-\mathbf{k}_{p} \cdot \mathbf{v}^{\prime}\right) s_{1}} e^{-\left(i \omega_{0}+\gamma_{e g}\right)\left(s_{2}-s_{1}\right)} \times\left\langle\Omega_{f}\left(t_{3}\right) \Omega_{p}^{*}\left(t_{2}-\tau\right) \Omega_{b}\left(t_{1}\right) \Omega_{f}^{*}\left(s_{3}\right) \Omega_{b}^{*}\left(s_{2}\right) \Omega_{p}\left(s_{1}-\tau\right)\right\rangle .
\end{aligned}
$$

It is important to remark that the presence of a sixth-order field correlation product is a reflection of the fact that the same fields act over an extended spatial region, enabling them to induce long-range spatial correlations within the sample. These long-range correlations between spatially separated atoms are then degraded by the independent thermalizing actions of dephasing collisions and spontaneous emission at each spatial location. In this sense the field statistics act differently from the collisional dephasing and spontaneous emission terms. We note, that in contrast to the above development, the conventional Maxwell-Bloch treat- ment, by focusing on the macroscopic polarization induced at a general location within the sample, implies an independent average over the two factors in Eq. (1) and therefore does not correctly take into account the higher-order correlations [17].

In order to make further progress we make the simplifying assumption that the incident-field amplitudes are jointly circular complex random Gaussian variables. Expressing the incident fields in terms of their associated Rabi frequencies $\Omega_{i}=\mu_{e g} E_{i} / \hbar$, where $\mu_{e g}$ is the dipole matrix element of the transition (we assume that $\mu_{e g}=\mu_{e^{\prime} g}$ ) and $E_{i}$ is the ampli- 
tude of the $i$ th incident field $(i=f, b$, or $p$ ), we further assume for convenience a chaotic field model for the incident fields having a Lorentzian spectrum of full width at half maximum $2 B$. Hence the Rabi frequencies of the incident fields are taken to possess a zero mean value and a secondorder correlation function given by

$$
\left\langle\Omega_{i}(t) \Omega_{j}^{*}\left(t^{\prime}\right)\right\rangle=C_{i j} \exp \left[-2 B_{i j}\left|t-t^{\prime}\right|\right]
$$

The power contained in the field $i$ is proportional to $C_{i i}$. This model is a reasonable representation of multimode lasers dominated by technical noise [6] and allows us to obtain analytical results. We can then invoke the Gaussian deconvolution theorem [18] to express the sixth-order field correlations as products of second-order field correlations:

$$
\begin{array}{r}
\left\langle\Omega\left(t_{1}\right) \Omega\left(t_{2}\right) \cdots \Omega\left(t_{n}\right) \Omega *\left(t_{n+1}\right) \cdots \Omega *\left(t_{2 n}\right)\right\rangle \\
=\sum\left\langle\Omega\left(t_{i}\right) \Omega *\left(t_{j}\right)\right\rangle\left\langle\Omega\left(t_{k}\right) \Omega *\left(t_{1}\right)\right\rangle \cdots,
\end{array}
$$

where the sum is over all possible different pairings. Each of the sixth-order field correlations thereby generates six different products of three second-order correlation functions leading to a total of 96 different integrals to evaluate. These second-order correlation functions effectively limit the time separations permissible between the field interactions responsible for the evolution of the coherences $\rho_{e^{\prime} g}^{f, p^{*}, b}$ and $\left[\rho_{e^{\prime} g}^{f, p^{*}, b}\right]^{*}$ in Eq. (1). One can graphically represent the resulting contributions through what we call double-sided Liouville diagrams (essentially equivalent to the "factored time correlation diagrams" of Ref. [14]).

Figure 3 displays six such diagrams for the case in which the evolutions of the coherences $\rho_{e^{\prime} g}^{f, p^{*}, b}$ and $\left[\rho_{e^{\prime} g}^{f, p^{*}, b}\right]^{*}$ both follow pathway I. The six different diagrams correspond to the six different permutations of the Gaussian decomposition of the sixth-order correlation product into products of second-order correlations. The shaded bubbles schematically represent the time intervals over which there exists significant correlation between two incident fields as specified by Eq. (3). Note that the "decoherence" introduced by the limited field correlations acts differently from that due to dephasing collisions (or spontaneous emission). As mentioned above, the incident fields act over an extended spatial region, thereby introducing correlations between spatially separated atomic coherences, which are thus able to generate a coherent signal field. This is manifested by the fact that at least one second-order field correlation involves interactions on both sides of the double-sided Liouville diagrams, as shown in Fig. 3. The finite correlation time of the fields temporally "localizes" the respective field interactions in that it restricts the allowed temporal separations between the moments at which the field acts. However, this field coherence temporal localization effect is indifferent to the sense of time propagation, depending only on the separation in time between the respective field interactions (a quality that the authors of Ref. [14] describe as a two-sided time symmetry).
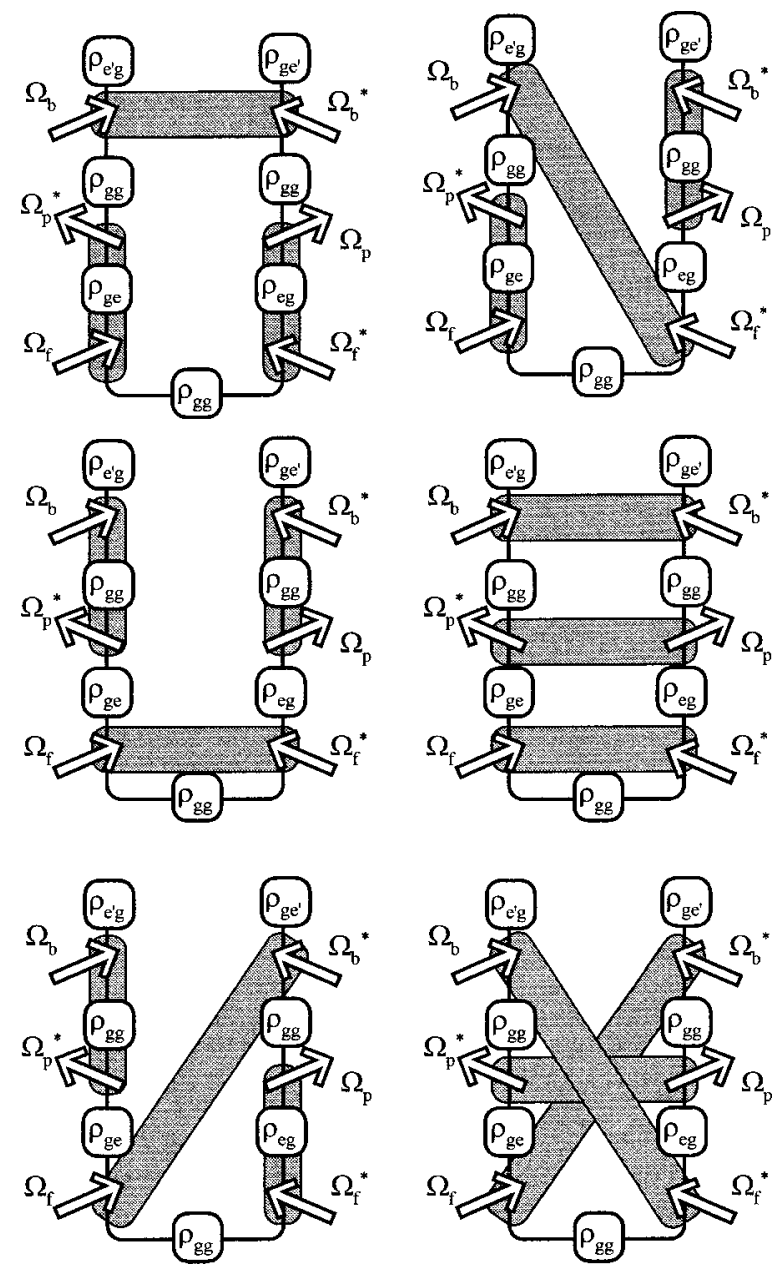

FIG. 3. Double-sided Liouville diagrams corresponding to the case for which both $\rho_{e^{\prime} g}^{f, p^{*}, b}$ and $\left[\rho_{e^{\prime} g}^{f, p^{*}, b}\right]^{*}$ follow pathway I. The six different diagrams result from using the Gaussian decorrelation theorem to write the sixth-order field correlation in terms of the six possible permutations of triple products of second-order field correlations.

In contrast, the atomic coherence decay terms $\gamma_{e g}$ and $\gamma_{e^{\prime} e}$ act solely along a single branch of the double Liouville diagrams (i.e., along the individual density matrix evolution paths). Moreover, the atomic decay terms always commence at a specific time, given by the instant at which the appropriate field interaction occurs, and propagate along the direction of increasing time (one-sided time symmetry).

In order to simplify the resulting integrals we assume that all field amplitudes are slowly varying compared to atomic decay rates and field correlation times allowing us to consider the field correlation coefficients $C_{i j}$ to be essentially constant over the time scales of interest. The 96 different integrals can be reduced to 24 prototypical integrals by using a short hand notation in which the pump-field Rabi frequencies are denoted by the pairs $\left(\Omega_{j}, \Omega_{k}\right)$ and $\left(\Omega_{m}^{*}, \Omega_{n}^{*}\right)$ for the interactions building up $\rho_{e^{\prime} g}^{f, p^{*}, b}$ and $\left[\rho_{e^{\prime} g}^{f, p^{*}, b}\right]^{*}$, respectively. Here the subscript $j(m)$ can stand for either the forward pump $f$ or the backward-propagating pump $b$, with the index $k(n)$ taking on the other value. These 24 prototypical inte- 


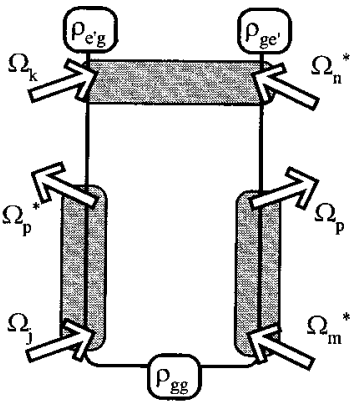

(a)

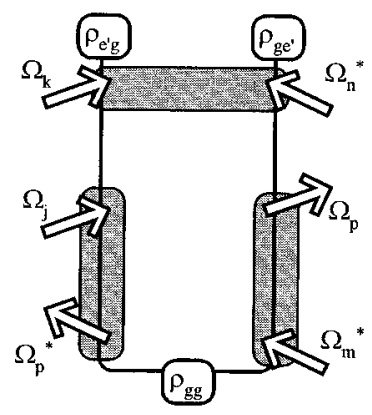

(c)

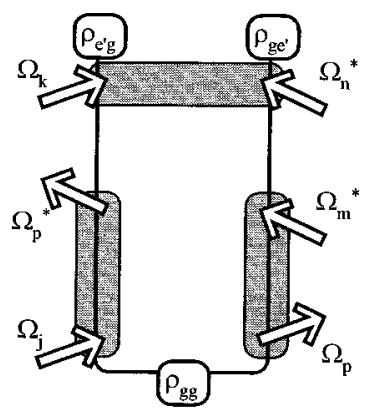

(b)

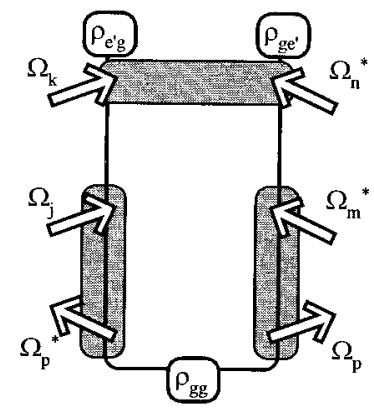

(d)
FIG. 4. Some of the 24 prototypical double-sided Liouville diagrams grouped according to the particular combination of secondorder correlations, shown schematically by the shaded bubbles. The subscript pairs $(j, k)$ and $(m, n)$ can stand for either $(f, b)$ or $(b, f)$; thus each diagram actually represents four different cases.

grals can be further grouped into six sets of four different six-dimensional integrals according to the type of secondorder correlation present. The double-sided Liouville diagrams corresponding to these six sets are shown in Figs. 4-9.

Given the above approximations, one can, with some patience, analytically carry out the resulting integrations for these 24 prototypical integrals. In doing so it is helpful to use the identity

$$
\exp \left[-B\left|t-t^{\prime}\right|\right]=\frac{B}{\pi} \int_{-\infty}^{\infty} d u \frac{\exp \left[i u\left(t-t^{\prime}\right)\right]}{u^{2}+B^{2}}
$$

Expanding the resulting expressions in powers of $(1 / \Delta)$, the lowest-order contribution varies as $(1 / \Delta)^{4}$. This should be contrasted with the $(1 / \Delta)^{6}$ dependence that would have resulted if the incident fields were monochromatic. At this level there are several different cancellations that dramatically reduce the number of terms one must calculate.

Consider first any pair of double-sided Liouville diagrams (with the second-order field correlations included) that differ simply by the exchange in the time order of the action of one of the pump waves and the probe wave. As an example, the integrals corresponding to the diagrams of Figs. 4(a) and 4(c) differ simply by the order in which the pump wave $\Omega_{j}$ and the probe wave $\Omega_{p}$ act on the $\rho_{e^{\prime} g}$ side of the double Liouville diagram. Apart from the inclusion of a bandwidth term, the difference between these two diagrams is entirely equivalent to the difference between the contributions of paths I and

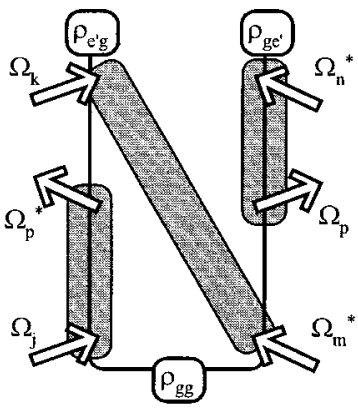

(a)

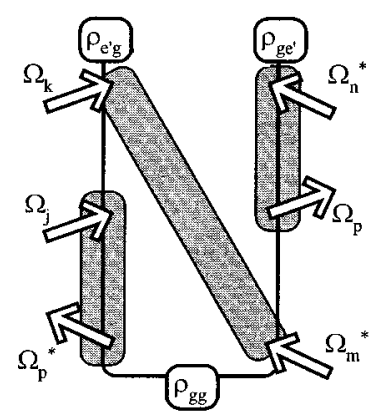

(c)

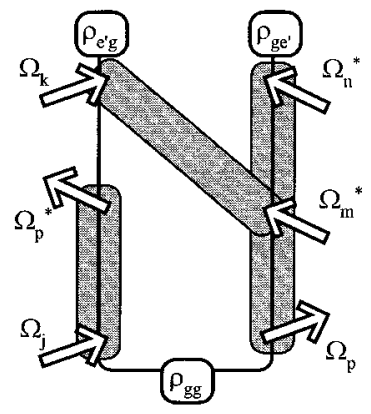

(b)

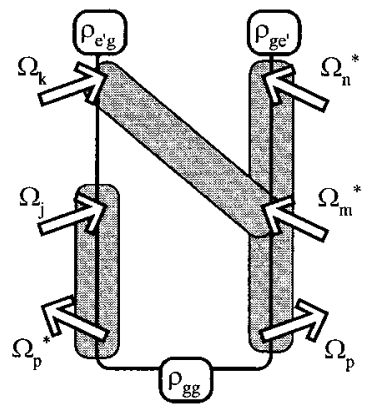

(d)
FIG. 5. Same as Fig. 4.

II to the third-order density matrix element. At the order $(1 / \Delta)^{4}$ these two separate pathways interfere destructively with one another. This same pairwise cancellation also occurs for the diagrams of Fig. 4(c) and 4(d), as well as for the pairs of contributions represented by diagrams of Figs. 5(a) and 5(b), Figs. 5(c) and 5(d), Figs. 8(a) and 8(b), and Figs.

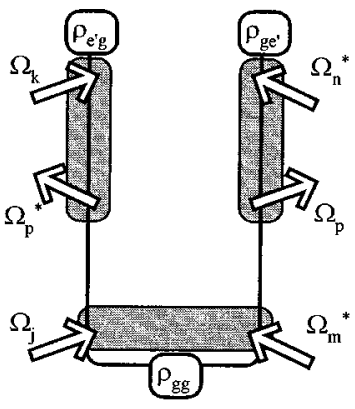

(a)



(c)

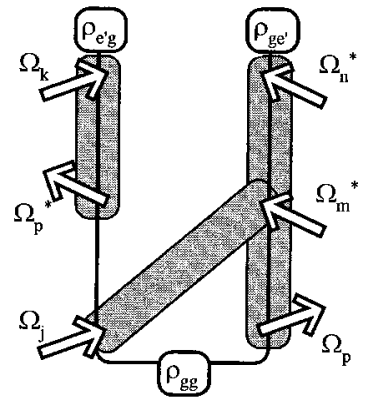

(b)

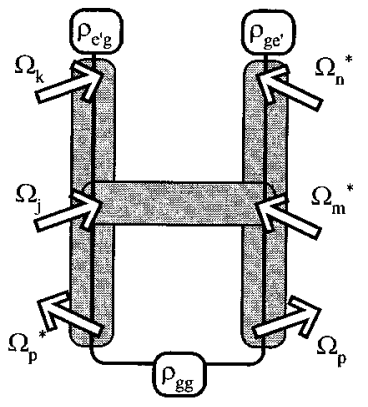

(d)
FIG. 6. Same as Fig. 4. 




(a)

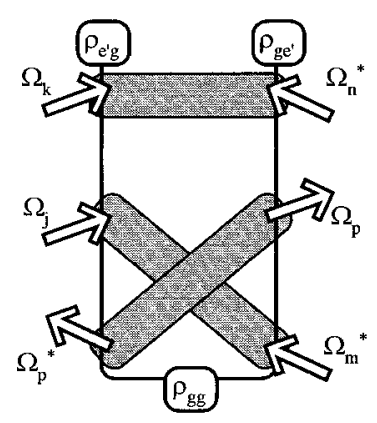

(c)

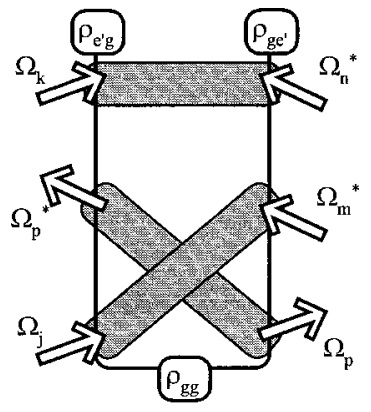

(b)

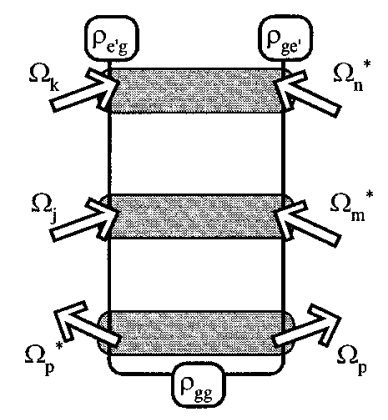

(d)

FIG. 7. Same as Fig. 4.

$8(\mathrm{c})$ and $8(\mathrm{~d})$. This reduces by half the number of integrals one must calculate.

With patience the other integrals can all be calculated. To lowest order in $(1 / \Delta)$, the dominant contributions come from portions of the integrals represented in Figs. 6(a) and 7(a). The contribution from Fig. 6(a) is given by

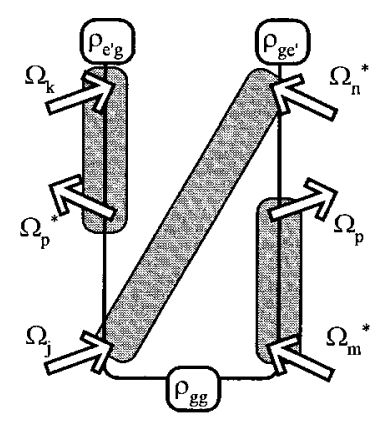

(a)

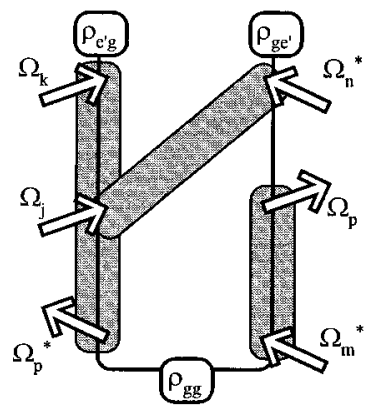

(c)

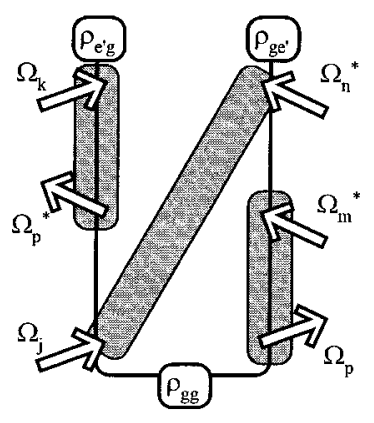

(b)

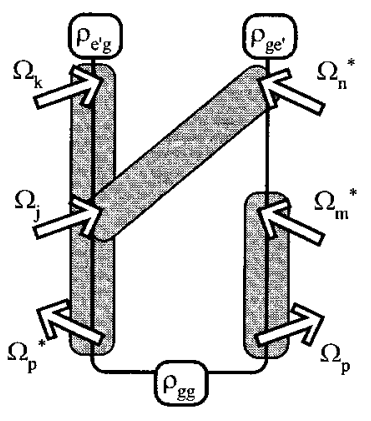

(d)

FIG. 8. Same as Fig. 4.

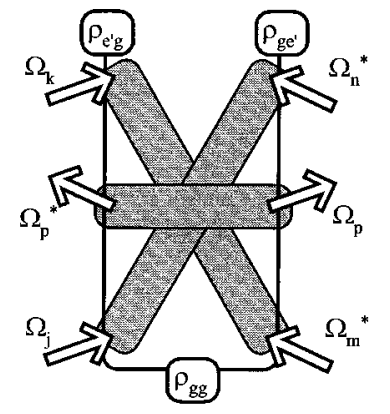

(a)

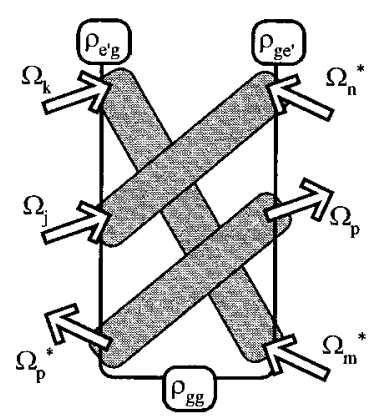

(c)

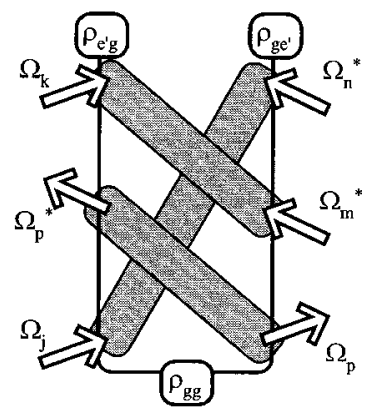

(b)

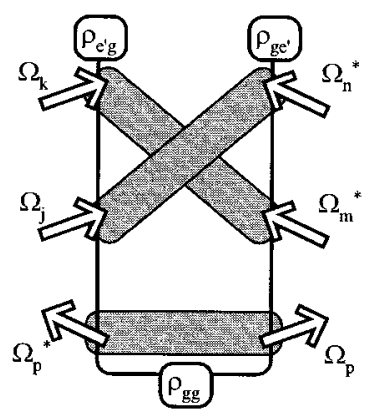

(d)
FIG. 9. Same as Fig. 4.

$$
\begin{aligned}
S_{6 \mathrm{a}} \sim & \frac{\left|\Omega_{f}\right|^{2}\left|\Omega_{p}\right|^{2}\left|\Omega_{b}\right|^{2}}{\Delta^{4}} e^{-i \mathbf{k}_{s} \cdot\left(\mathbf{v}-\mathbf{v}^{\prime}\right) t} C_{j m} C_{k p} C_{p n} \\
& \left.\left.\times \frac{2 B_{j m} e^{-\left(B_{m p}+B_{p n}\right)|\tau|}}{\left[i \mathbf{k}_{s} \cdot\left(\mathbf{v}-\mathbf{v}^{\prime}\right)+2 \gamma_{e g}\right]}\right] \frac{1}{i \mathbf{k}_{j} \cdot \mathbf{v}-i \mathbf{k}_{m} \cdot \mathbf{v}^{\prime}+2 \gamma_{e g}}\right] \\
& \times\left[\frac{1}{i \mathbf{k}_{s} \cdot \mathbf{v}-i \mathbf{k}_{m} \cdot \mathbf{v}^{\prime}+2 \gamma_{e g}}+\frac{1}{i \mathbf{k}_{m} \cdot \mathbf{v}-i \mathbf{k}_{s} \cdot \mathbf{v}^{\prime}+2 \gamma_{e g}}\right]
\end{aligned}
$$

while that from Fig. 7(a) is given by

$$
\begin{aligned}
S_{7 \mathrm{a}} \sim & \frac{\left|\Omega_{f}\right|^{2}\left|\Omega_{p}\right|^{2}\left|\Omega_{b}\right|^{2}}{\Delta^{4}} e^{-i \mathbf{k}_{s} \cdot\left(\mathbf{v}-\mathbf{v}^{\prime}\right) t} C_{j m} C_{p p} C_{k n} \\
& \times\left[\frac{2 B_{j m}}{i \mathbf{k}_{s} \cdot\left(\mathbf{v}-\mathbf{v}^{\prime}\right)+2 \gamma_{e g}}\right]\left[\frac{1}{i \mathbf{k}_{j} \cdot \mathbf{v}-i \mathbf{k}_{m} \cdot \mathbf{v}^{\prime}+2 \gamma_{e g}}\right] \\
& \times\left[\frac{1}{i \mathbf{k}_{s} \cdot \mathbf{v}-i \mathbf{k}_{m} \cdot \mathbf{v}^{\prime}+2 \gamma_{e g}+B_{k n}+B_{p p}}\right. \\
& \left.+\frac{1}{i \mathbf{k}_{m} \cdot \mathbf{v}-i \mathbf{k}_{s} \cdot \mathbf{v}^{\prime}+2 \gamma_{e g}+B_{k n}+B_{p p}}\right]
\end{aligned}
$$

Recall that the pairs $(j, k)$ and $(m, n)$ can stand for either $(f, b)$ or $(b, f)$ so that each of the above expressions represents four different possible terms. It remains to integrate over the velocity distribution for $\mathbf{v}$ and $\mathbf{v}^{\prime}$.

Both of the expressions in Eqs. (5) and (6) are proportional to the bandwidth of the incident radiation fields $B$, 
allowing us to identify the signal as being "fluctuation" induced. Interestingly, all of the resonances involve field interactions on both sides of the double Liouville diagram, i.e., they are resonances between field-induced dipoles at spatially separated points as is evident from the presence of both $\mathbf{v}$ and $\mathbf{v}^{\prime}$ in every denominator. None of these terms would have appeared if the two-point spatial correlation of Eq. (1) had been factorized. In contrast to the above contributions, PIER4 terms arise from collisional destruction of interferences between pathways III and IV and involve a resonance associated with the interactions along a single side of the double Liouville diagram. These resonances appear only in order $(1 / \Delta)^{6}$ and are negligible under our conditions.

We find that the choice of a far-impact detuning effectively breaks the symmetry between the many possible coherent pathways. Essentially the time-order diagrams in which the counter-rotating probe wave acts second are favored, since one can then form stimulated Rayleigh scattering resonances between it and the pump beams involved in either the first or third interaction. Thus, only the timeordered pathways I and III contribute to lowest order $(1 / \Delta)^{4}$. Among the multitude (48) of double-sided Liouville diagrams generated by these two pathways, the diagrams in which there exists a correlation between the first interactions on either side of the double Liouville diagram are favored. We believe this is a result of the rather large detuning in the far impact regime, which, according to an uncertainty principle argument, creates an initial coherence that exists for a time of order $1 / \Delta$. The field correlation between these interaction helps to "synchronize" the creation of the two coherences at the spatially distinct points $\mathbf{r}$ and $\mathbf{r}^{\prime}$ of Eq. (1). Of all the diagrams in Figs. 6, 7, and 9, only those of Figs. 6(a) and 7 (a) meet both these requirements.

\section{EFFECTS OF THE ATOMIC MOTION}

The atomic motion greatly influences the magnitude of the phase-conjugate signal in the far impact regime, effectively determining the strength of the scattering resonances in the denominators of Eqs. (5) and (6). This dependence was neglected by Bratfalean and Ewart [15], who assumed that the signal should be independent of the atomic motion if the incident fields were detuned by many Doppler widths. This is actually true, however, only in the extreme limit where the homogeneous broadening dominates the linewidth. Since the signal is proportional to a two-point spatial correlation function (in the limit of a thin nonlinear medium), it is important that the atomic coherences created at spatially separated locations continue to maintain the correlations impressed upon them by the action of the incident fields. These correlations can be destroyed by phase-changing collisions, spontaneous emission, or a Doppler dephasing of the two spatially separated coherences. Interestingly, for the present case of detunings in the far impact region, population gratings created by the spatial interference between one of the pump beams and the probe seem to play no role, at order $1 / \Delta^{4}$, as can be readily seen by noting that Eqs. (5) and (6) contain no reference to the population decay rates $\gamma_{e e}$ or $\gamma_{g g}$ but rather involve only the coherence decay rate $\gamma_{e g}$.

The dephasing induced by the relative motion between the

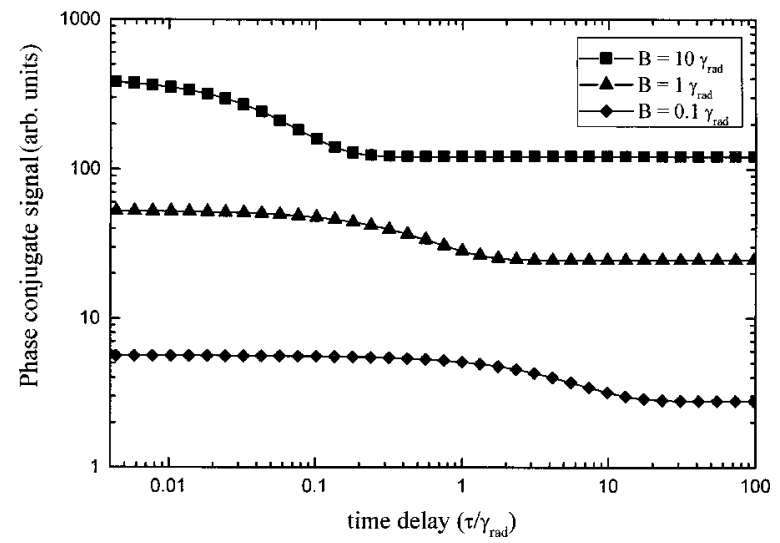

FIG. 10. The phase-conjugate signal shown as a function of the time delay of the incident probe field. Here the atomic decay parameters are $\gamma_{\mathrm{inh}}=50 \gamma_{\mathrm{rad}}$ and $\gamma_{\mathrm{col}}=5 \gamma_{\mathrm{rad}}$. Three different bandwidths for the incident field are shown, $B=(10,1,0.1) \gamma_{\mathrm{rad}}$.

coherences generated at the spatially separated points $\mathbf{r}$ and $\mathbf{r}^{\prime}$ causes the denominators of Eqs. (5) and (6) to contain resonances (i) when $\mathbf{k}_{s} \cdot\left(\mathbf{v}-\mathbf{v}^{\prime}\right)=0$, i.e., when the projection of the two atomic velocities along the signal wave vector is the same, (ii) when $\mathbf{k}_{j} \cdot \mathbf{v}=\mathbf{k}_{m} \cdot \mathbf{v}^{\prime}$, i.e., when the projection of the atomic velocities along the wave vector of the first interacting field is the same for the two first-order coherences, (iii) when $\mathbf{k}_{s} \cdot \mathbf{v}=\mathbf{k}_{m} \cdot \mathbf{v}^{\prime}$ and/or $\mathbf{k}_{m} \cdot \mathbf{v}=\mathbf{k}_{s} \cdot \mathbf{v}^{\prime}$, i.e., when the projection of the atomic velocity along the wave vector of the first interacting field is the same as the projection of the other atomic velocity on the signal wave vector. To determine the overall signal strength it remains to integrate over the Maxwell-Boltzmann distribution of velocities $\mathbf{v}$ and $\mathbf{v}^{\prime}$. This is done in the Appendix for the case in which the angle $\theta$ between the forward-going pump wave and the probe wave (see Fig. 1) is small and for which all three incident fields originate from the same source. Assuming the same bandwidth $B$ for all possible second-order field correlations, the result can be schematically written as

$$
S \sim \frac{2 B\left|\Omega_{f}\right|^{2}\left|\Omega_{p}\right|^{2}\left|\Omega_{b}\right|^{2}}{\Delta^{4}}\left[\sum_{i=1}^{4}\left(e^{-2 B|\tau|} F_{6 \mathrm{a}}^{i}+F_{7 \mathrm{a}}^{i}\right)\right] .
$$

Specific expressions for the different contributions $F_{6 \mathrm{a}}^{i}$ and $F_{7 \mathrm{a}}^{i}$ can be found in the Appendix.

We show in Fig. 10 the phase-conjugate signal normalized to the bandwidth as a function of the time delay between the probe and pump fields for three different bandwidths. Note that, apart for an overall multiplicative factor, the contribution of $S_{6 \mathrm{a}}$ at zero delay is independent of the bandwidth of the incident radiation. At long enough time delays $(B|\tau| \gg 1)$, the signal reduces to a constant background that is made up solely of the contributions of $S_{7 \mathrm{a}}$. Apart from the overall multiplicative factor of the bandwidth, the contributions of this term tend to decrease with increasing bandwidth.

This is more evident in Fig. 11, where we have plotted the signal "contrast," i.e., the ratio of the zero-delay peak to the long-time-delay background, as a function of the dipole dephasing rate $\gamma_{e g}$, for several different bandwidths. The common overall multiplicative factor of the bandwidth can- 


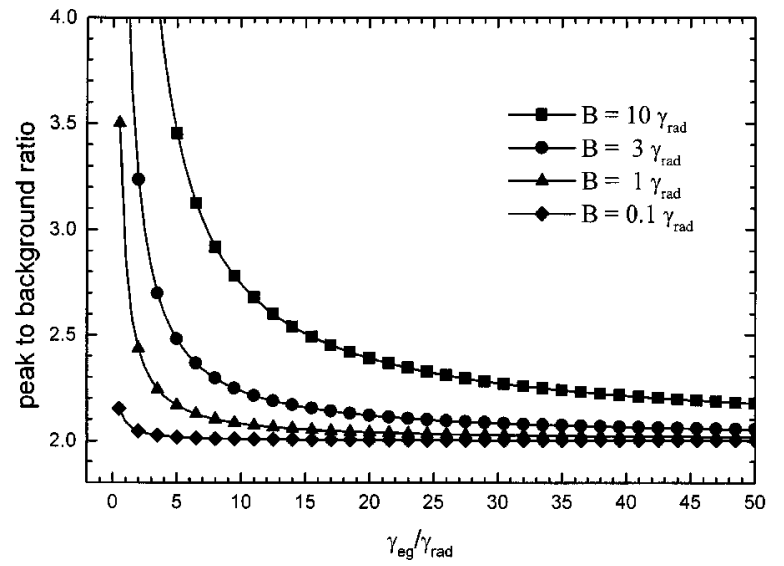

FIG. 11. The contrast between the zero-time-delay peak and the long-time-delay constant background plotted as a function of the dipole dephasing rate $\gamma_{e g}$, for several different bandwidths of the incident radiation fields. Here the inhomogeneous width $\gamma_{\text {inh }}=50 \gamma_{\text {rad }}$.

cels upon forming this ratio, so that the contrast is a more sensitive measure of the interplay between the atomic "memory time" limited by the ground to excited state coherence dephasing time $\gamma_{e g}$, and the finite coherence time of the incident fields as determined by their bandwidth $B$. One can qualitatively understand the trend shown in Fig. 11 by referring to the double-sided Liouville diagrams of Figs. 6(a) and 7(a). The difference between these two figures is that the last two correlations of Fig. 7(a) are between the dipoles $\rho_{e^{\prime} g}^{f, p^{*}, b}$ and $\left[\rho_{e^{\prime} g}^{f, p^{*}, b}\right]^{*}$ on opposite sides of the double-sided Liouville diagram, whereas in Fig. 6(a) the last two field correlations are between interactions occurring within the same active atom. Remembering that the width of the correlation bubbles along the time (vertical) axis varies inversely with the bandwidth, one realizes that a relatively large bandwidth forces the second and third field interactions on the left and right sides of Fig. 7(a) to be nearly coincident. In contrast, this field-induced simultaneity is not present for the diagram of Fig. 6(a), where the second and third field interactions can effectively be translated relative to one another along the time axis. This extra freedom leads to a larger contribution from Fig. 6(a) when the bandwidth becomes large relative to the radiative decay rate. Of course the permissible time separations between interactions along the time line of a single dipole are still limited by its memory time. Thus for a large dipole dephasing rate $\left(\gamma_{e g} \gg B\right)$, the extra constraint imposed by the bandwidth on Fig. 7(a) matters relatively little and the contrast varies only slightly as a function of the bandwidth.

The conventional interpretation for the four-wave-mixing signal arising from our choice of pump polarizations would be as follows. The forward-going pump interferes with the incident probe wave to form a spatially modulated intensity pattern that in turn creates a spatial modulation of the complex susceptibility within the active medium. This susceptibility grating then diffracts a portion of the backward-going pump wave, forming the phase-conjugate signal. Such an interpretation clearly favors the contribution from the timeordered pathway II of Fig. 2 relative to that of pathway IV. In addition, one might suspect that pathway II has an extra advantage since, in building up the third-order dipole $\rho_{e^{\prime} g}^{f, p^{*}, b}$, this pathway passes through the intermediate state $\rho_{g g}^{f, p^{*}}$, a state that does not suffer from either radiative decay or collisional dephasing. In contrast, pathway IV passes through the excited-state coherence $\rho_{e^{\prime},}^{p^{*}, b}$, which suffers from both of these decay rates. Naively, then, one might expect that the contributions from case $1(j=m=f, k=n=b)$ which corresponds to placing pathway II on both sides of the doublesided Liouville diagram, would dominate over the others. Surprisingly, we find that just the opposite occurs; for relatively small dipole dephasing rates $\left(\gamma_{e g} \lesssim 10 \gamma_{\text {rad }}\right)$, the signal is dominated by contributions from case 4 , i.e., when $(j$ $=m=b, k=n=f$ ), corresponding to the time-ordered pathway IV on both sides of the double-sided Liouville diagram. To understand why, recall that for our case of a far-impact detuning the resonant denominators all include field interactions creating induced dipole moments on opposite sides of the double-sided Liouville diagram. The scattering resonances occur (i) between the two third-order dipoles $\rho_{e^{\prime} g}^{f, p^{*}, b}(\mathbf{r}, \mathbf{v})$ and $\left[\rho_{e^{\prime} g}^{f, p^{*}, b}\left(\mathbf{r}^{\prime}, \mathbf{v}^{\prime}\right)\right]^{*}$ occurring when $\mathbf{k}_{s} \cdot(\mathbf{v}$ $\left.-\mathbf{v}^{\prime}\right)=0$, (ii) between the two first-order dipoles $\rho_{x g}^{j}(\mathbf{r}, \mathbf{v})$ and $\left[\rho_{x g}^{m}\left(\mathbf{r}^{\prime}, \mathbf{v}^{\prime}\right)\right]^{*}\left(x\right.$ being either $e$ or $e^{\prime}$ depending on whether the interaction is with the forward- or backwardgoing pump wave) occurring when $\mathbf{k}_{j} \cdot \mathbf{v}=\mathbf{k}_{m} \cdot \mathbf{v}^{\prime}$, and (iii) between the first-order dipole on one side of the double-sided Liouville diagram and the third-order dipole on the other side, i.e., when $\mathbf{k}_{s} \cdot \mathbf{v}=\mathbf{k}_{m} \cdot \mathbf{v}^{\prime}$ and $\mathbf{k}_{m} \cdot \mathbf{v}=\mathbf{k}_{s} \cdot \mathbf{v} \cdot$. Case 4 is the only case for which all of the resonances occur for roughly the same velocity class provided the angle $\theta$ between the forward-going pump and probe waves is small. Hence case 4 dominates in the limit of strong Doppler broadening. As the dipole dephasing rate $\gamma_{e g}$ increases, homogeneous broadening becomes more important and the contribution of the other cases becomes significant.

\section{CONCLUSIONS}

In summary, we have presented a concise analytical model for time-delayed four-wave mixing in the far impact regime that has allowed us to probe the interplay between the correlation time associated with the incident fields and atomic memory time. For large detunings we found that the signal is dominated by correlations between dipole moments induced at spatially separated points within the active medium, correlations that are not treated properly by the usual Maxwell-Bloch equation formulation of four-wave mixing. Indeed, our result in some sense maximally violates the Maxwell-Bloch treatment since at lowest order no part of the signal is factorizable into the product of contributions from single atoms. Introducing double Liouville diagrams in order to take into account these correlations, we were able to show, in the case of fields modeled adequately by a circular complex random Gaussian variable, that the signal arises mainly due to a small subset of eight out of a total of 96 possible diagrams. The contrast between the zero-delay peak and the long-time background signal in a time-delayed four-wavemixing experiment was found to depend sensitively on the 
bandwidth of the incident fields when the collisional dephasing rate is much smaller than the inhomogeneous Doppler width of the active atoms.

In order to arrive at these conclusions we have made several simplifying assumptions. Transit time effects have been ignored, meaning that our results are valid only for a nonlinear medium that is thin. More severe is our modeling of the incident field as a single broadband field; most pulsed-dye lasers typically oscillate in several longitudinal modes. It is clearly possible to generalize the above treatment to account for several different longitudinal modes. In fact, we believe this to be an interesting way to study the cross correlations between longitudinal modes in a pulsed-dye laser. The application of this basic formalism to the case of a two-mode field will be presented in a subsequent publication.

In summary, we have shown that the effect of the finite bandwidth of the stochastic field has far richer role than that of collisional dephasing. Field correlations are able to act both locally at an individual dipole and at spatially separated points. Indeed, it is the correlations, induced by the field, between spatially separated atoms that are responsible for the four-wave-mixing signal in the far impact regime. We have shown that, most surprisingly, in the case of far-impact detunings, the dominant terms responsible for the signal possess nonlocal resonances that involve field interactions between spatially separated atoms. On the other hand, PIERtype resonances, which appear at order $1 / \Delta^{6}$, involve only resonances in single atoms that are excited coherently within the interaction region. Thus, in contrast to the PIER-type resonances, these nonlocal resonances cannot be described by an effective nonlinear susceptibility for the medium.

\section{ACKNOWLEDGMENTS}

The authors are grateful to the British Council for partial funding in support of the collaboration involved in this work. M.B. would like to thank José Carlos Viana Gomes for many stimulating discussions and the Ministerio da Ciência e da Tecnologia (Portugal) for partial financial support. M.K. is grateful to the Royal Society (U.K.) for personal financial support.

\section{APPENDIX}

In this Appendix we briefly indicate how to carry out the averaging over the Maxwell-Boltzmann velocity profiles for $\mathbf{v}$ and $\mathbf{v}^{\prime}$ in Eqs. (5) and (6). We treat the case of a classic phase-conjugate geometry for the incident beams (see Fig. 1) and assume that the angle $\theta$ between the probe and forwardgoing pump beam is small in the sense that $\sin \theta \approx \theta$. There exist four separate cases to consider for each of Eqs. (5) and (6), depending on the choice of pump beams for the fields $j$, $k, m$, and $n$. It is convenient to use a coordinate system with the $x$ axis aligned along the incident probe beam, i.e., $\mathbf{k}_{p}$ $=k \hat{\mathbf{x}}$. Then, using the fact that $\theta$ is small, $\mathbf{k}_{f} \approx k(\hat{\mathbf{x}}-\theta \hat{\mathbf{y}})$, while $\mathbf{k}_{b}=-\mathbf{k}_{f}$ and $\mathbf{k}_{s}=-\mathbf{k}_{p}$. If we assume that the incident beams exist for a time long compared to the inverse of the inhomogeneous width then the signal integrated over the entire pulse width contains a factor

$$
\int_{-T / 2}^{T / 2} e^{-i \mathbf{k}_{s} \cdot\left(\mathbf{v}-\mathbf{v}^{\prime}\right) t} d t \rightarrow 2 \pi \delta\left[\mathbf{k}_{\mathbf{s}} \cdot\left(\mathbf{v}-\mathbf{v}^{\prime}\right)\right] .
$$

This simplification can be interpreted as requiring the projection of the velocity along the direction of propagation of the signal wave to be equal for the third-order dipoles induced at the spatially separated points $\mathbf{r}$ and $\mathbf{r}^{\prime}$. Otherwise, neglecting velocity-changing collisions, over the duration of the pulse there would be a Doppler dephasing between the fields emitted by the two dipoles, leading to a destructive cancellation of the contribution of the pair. We now go on to consider explicitly the four different time orderings of the pump-field interactions for the contributions of Eq. (6). The corresponding contributions for Eq. (5) can then be readily obtained by taking the limit of $B \rightarrow 0$ of the respective results.

\section{Case 1: $j=m=f, k=n=b$}

For this case the velocity-dependent denominators of Eq. (6) take the form

$$
\begin{aligned}
F_{7}^{1}\left(\mathbf{v}, \mathbf{v}^{\prime}\right)= & \frac{1}{\left[-i k\left(v_{x}-v_{x}^{\prime}\right)+2 \gamma_{e g}\right]} \\
& \times\left[\frac{1}{i k\left(v_{x}-v_{x}^{\prime}\right)-i k \theta\left(v_{y}-v_{y}^{\prime}\right)+2 \gamma_{e g}}\right] \\
& \times\left[\frac{1}{-i k\left(v_{x}+v_{x}^{\prime}\right)+i k \theta v_{y}^{\prime}+2\left(\gamma_{e g}+B\right)}\right. \\
& \left.+\frac{1}{i k\left(v_{x}+v_{x}^{\prime}\right)-i k \theta_{v y}+2\left(\gamma_{e q}+B\right)}\right] .
\end{aligned}
$$

Each of the independent velocity components $v_{x}, v_{x}^{\prime}, v_{y}$ and $v_{y}^{\prime}$, must now be integrated over the Maxwell-Boltzmann distribution

$$
\frac{1}{u \sqrt{u}} \int_{-\infty}^{\infty} d v \exp \left[-v^{2} / u^{2}\right]
$$

where $u=\sqrt{2 k_{B} T / M}$ is the most probable speed of the active atoms with mass $M ; k_{B}$ is Boltzmann's constant. Using the $\delta$ function of Eq. (A1) to integrate over $v_{x}^{\prime}$, one readily obtains

$$
\begin{aligned}
\left\langle F_{7}^{1}\right\rangle= & \frac{2}{\pi\left(2 \gamma_{e q}\right) k u} \int_{-\infty}^{\infty} d\left(\frac{v_{x}}{u}\right) \int_{-\infty}^{\infty} d\left(\frac{v_{y}}{u}\right) \int_{-\infty}^{\infty} d\left(\frac{v_{y}^{\prime}}{u}\right) \\
& \times \exp -\left[\left(2 v_{x}^{2}+v_{y}^{2}+v_{y}^{\prime 2}\right) / u^{2}\right] \\
& \times\left(\frac{1}{-i k \theta\left(v_{y}-v_{y}^{\prime}\right)+2 \gamma_{e g}}\right) \\
& \times\left[\frac{1}{-2 i k v_{x}+i k \theta v_{y}^{\prime}+2\left(\gamma_{e q}+B\right)}\right. \\
& \left.+\frac{1}{2 i k v_{x}-i k \theta v_{y}+2\left(\gamma_{e g}+B\right)}\right] .
\end{aligned}
$$

The integral over $v_{x}$ can be written in terms of the error 
function of complex argument [19]

$$
W(z)=\frac{1}{i \pi} \int_{-\infty}^{\infty} d t \frac{\exp \left(-t^{2}\right)}{t-z} \text { with } \operatorname{Im}(z)>0,
$$

yielding

$$
\begin{aligned}
\left\langle F_{7}^{1}\right\rangle= & \frac{1}{\left(2 \gamma_{e g}\right)(k u)^{2}} \int_{-\infty}^{\infty} d\left(\frac{v_{y}}{u}\right) \int_{-\infty}^{\infty} d\left(\frac{v_{y}^{\prime}}{u}\right) \\
& \times \exp -\left[\left(v_{y}^{2}+v_{y}^{\prime 2}\right) / u^{2}\right]\left(\frac{1}{-i k \theta\left(v_{y}-v_{y}^{\prime}\right)+2 \gamma_{e g}}\right) \\
& \times\left[W\left(\frac{-\theta v_{y}^{\prime}}{\sqrt{2} k u}+i \frac{\sqrt{2}\left(\gamma_{e g}+B\right)}{k u}\right)\right. \\
& \left.+W\left(\frac{\theta_{v y}}{\sqrt{2} k u}+i \frac{\sqrt{2}\left(\gamma_{e g}+B\right)}{k u}\right)\right] .
\end{aligned}
$$

Owing to the exponential terms the main contributions to the remaining integrals will come from the region close to $v_{y}$ $=0$ and $v_{y}^{\prime}=0$. Using the fact that the error function is slowly varying with respect to the real part of its argument near the origin, coupled with the angle $\theta$ being small, we expand the error functions as follows:

$$
W(z)=W(x+i y) \approx W(i y)+x \frac{\partial W}{\partial z}(z=i y) \quad \text { for }|x| \ll 1 .
$$

Here the first derivative of the error function of complex argument is [19]

$$
\frac{\partial W}{\partial z}(z=i y)=2 i\left[\frac{1}{\sqrt{\pi}}-y W(i y)\right] .
$$

To carry out the remaining integrals it is helpful to transform to new variables given by

$$
\begin{gathered}
\sigma=\frac{1}{\sqrt{2}} \frac{\left(v_{y}+v_{y}^{\prime}\right)}{u}, \\
f \eta=\frac{1}{\sqrt{2}} \frac{\left(v_{y}-v_{y}^{\prime}\right)}{u},
\end{gathered}
$$

leading to the final expression

$$
\begin{aligned}
\left\langle F_{7}^{1}\right\rangle= & \frac{\sqrt{2} \pi^{3 / 2}}{\left(2 \gamma_{e g}\right) \theta(k u)^{3}}\{W(i(\alpha+\beta)) W(i \alpha / \theta) \\
& \left.+\frac{\theta}{4} \frac{\partial W}{\partial z}[z=i(\alpha+\beta)] \frac{\partial W}{\partial z}(z=i \alpha / \theta)\right\} .
\end{aligned}
$$

Here we have defined the angles

$$
\alpha=\frac{\sqrt{2} \gamma_{e g}}{k u} \text { and } \beta=\frac{\sqrt{2} B}{k u} .
$$

The ratio $\alpha / \beta$ is thus a measure of the relative strength of the dipole dephasing rate (i.e., atomic memory) to the bandwidth of the incident fields. The contribution for the term of Eq. (5) for this case can be shown to be given by the same expression with $\beta$ set to zero.

\section{Case 2: $j=n=f, k=m=b$}

Using the same substitutions and transformations as above and performing the integral over $v_{x}^{\prime}$ using the $\delta$ function of Eq. (A1), the contribution for the velocity-dependent denominators of Eq. (6) is

$$
\begin{aligned}
\left\langle F_{7}^{2}\right\rangle= & \frac{2}{\pi\left(2 \gamma_{e g}\right) k u} \int_{-\infty}^{\infty} d\left(\frac{v_{x}}{u}\right) \int_{-\infty}^{\infty} d\left(\frac{v_{y}}{u}\right) \int_{-\infty}^{\infty} d\left(\frac{v_{y}^{\prime}}{u}\right) \\
& \times \exp -\left[\left(2 v_{x}^{2}+v_{y}^{2}+v_{y}^{\prime 2}\right) / u^{2}\right] \\
& \times\left(\frac{1}{2 i k v_{x}-i k \theta\left(v_{y}+v_{y}^{\prime}\right)+2 \gamma_{e g}}\right) \\
& \times\left[\frac{1}{-i k \theta v_{y}^{\prime}+2\left(\gamma_{e g}+B\right)}\right. \\
& \left.+\frac{1}{2 i k v_{x}-i k \theta v_{y}+2\left(\gamma_{e g}+B\right)}\right] .
\end{aligned}
$$

Using the algebraic identity $1 / A B=[1 /(B-A)](1 / A-1 / B)$ to rewrite the product of denominators containing $v_{x}$ and integrating over $v_{x}$ gives

$$
\begin{aligned}
\left\langle F_{7}^{\prime 2}\right\rangle= & \frac{1}{\left(2 \gamma_{e g}\right)(k u)^{2}} \int_{-\infty}^{\infty} d\left(\frac{v_{y}}{u}\right) \int_{-\infty}^{\infty} d\left(\frac{v_{y}^{\prime}}{u}\right) \\
& \times \exp -\left[\left(v_{y}^{2}+v_{y}^{\prime 2}\right) / u^{2}\right] \\
& \times\left\{\frac{1}{-i k \theta v_{y}^{\prime}+2\left(\gamma_{e g}+B\right)} W\left[\frac{\theta\left(v_{y}+v_{y}^{\prime}\right)}{\sqrt{2} u}+i \alpha\right]\right. \\
& +\frac{1}{i k \theta_{y}^{\prime}+2 B}\left(W\left[\frac{\theta\left(v_{y}+v_{y}^{\prime}\right)}{\sqrt{2} u}+i \alpha\right]\right. \\
& \left.\left.-W\left[\frac{\theta v_{y}}{\sqrt{2} u}+i(\alpha+\beta)\right]\right)\right\} .
\end{aligned}
$$

Expanding the error function about the point where the real part of the argument is zero and using the transformation of Eq. (A7) leads one to the expression 


$$
\begin{aligned}
\left\langle F_{7}^{2}\right\rangle= & \frac{\pi^{3 / 2}}{\left(2 \gamma_{e g}\right) \theta(k u)^{3}}\left\{W(i \alpha) W\left(i \frac{\sqrt{2}(\alpha+\beta)}{\theta}\right)+W\left(i \frac{\sqrt{2} \beta}{\theta}\right)\right. \\
& \times[W(i \alpha)-W(i \alpha+i \beta)]+\frac{\theta}{2 \sqrt{2}} \frac{\partial W}{\partial z}(z=i \alpha) \\
& \left.\times\left[\frac{\partial W}{\partial z}\left(z=i \frac{\sqrt{2}}{\theta}(\alpha+\beta)\right)-\frac{\partial W}{\partial z}\left(z=i \frac{\sqrt{2} \beta}{\theta}\right)\right]\right\} .
\end{aligned}
$$

To obtain the contribution of Eq. (5) for this case we once again set $\beta$ equal to zero. Using the values $W(0)=1$ and $(\partial W / \partial z)(0)=2 i / \sqrt{\pi}$, one finds

$$
\begin{aligned}
\left\langle F_{6}^{2}\right\rangle= & \frac{\pi^{3 / 2}}{\left(2 \gamma_{e g}\right) \theta(k u)^{3}}\left\{W(i \alpha) W\left(i \frac{\sqrt{2} \alpha}{\theta}\right)\right. \\
& \left.+\frac{\theta}{2 \sqrt{2}}\left(\frac{2 \sqrt{2} \alpha}{\theta}\right) W\left(i \frac{\sqrt{2} \alpha}{\theta}\right) \frac{\partial W}{\partial z}(z=i \alpha)\right\} .
\end{aligned}
$$

\section{Case 3: $j=n=b, k=m=f$}

For this case the resulting expression is formally equivalent to the complex conjugate of Eq. (A10). Since the result is real one obtains exactly the same results as for case 2 .

$$
\text { 4. Case 4: } j=m=b, k=n=f
$$

For this case, after integrating over $v_{x}^{\prime}$ using Eq. (A1), the contribution for the velocity-dependent denominators of Eq. (6) is

$$
\begin{aligned}
\left\langle F_{7}^{4}\right\rangle= & \frac{2}{\pi\left(2 \gamma_{e g}\right) k u} \int_{-\infty}^{\infty} d\left(\frac{v_{x}}{u}\right) \int_{-\infty}^{\infty} d\left(\frac{v_{y}}{u}\right) \int_{-\infty}^{\infty} d\left(\frac{v_{y}^{\prime}}{u}\right) \\
& \times \exp -\left[\left(2 v_{x}^{2}+v_{y}^{2}+v_{y}^{\prime 2}\right) / u^{2}\right]\left(\frac{1}{i k \theta\left(v_{y}-v_{y}^{\prime}\right)+2 \gamma_{e g}}\right) \\
& \times\left[\frac{1}{-i k \theta v_{y}^{\prime}+2\left(\gamma_{e g}+B\right)}+\frac{1}{+i k \theta v_{y}+2\left(\gamma_{e g}+B\right)}\right] .
\end{aligned}
$$

Integrating over $v_{x}$ and $v_{y}\left(v_{y}^{\prime}\right)$ for the first (second) term in parentheses one has

$$
\begin{aligned}
\left\langle F_{7}^{4}\right\rangle= & \frac{i \sqrt{2 \pi}}{\left(2 \gamma_{e g}\right) \theta^{2}(k u)^{3}} \int_{-\infty}^{\infty} d x e^{-x^{2}} \\
& \times\left[\frac{W(x+i \sqrt{2} \alpha / \theta)}{x+i(\sqrt{2} / \theta)(\alpha+\beta)}-\frac{W(-x+i \sqrt{2} \alpha / \theta)}{x-i(\sqrt{2} / \theta)(\alpha+\beta)}\right] .
\end{aligned}
$$

Expanding the error functions about the point $x=0$ up to terms linear in $x$ and integrating over $x$ yields

$$
\left\langle F_{7}^{4}\right\rangle=\frac{(2 \pi)^{3 / 2}}{\left(2 \gamma_{e g}\right) \theta^{2}(k u)^{3}}\left\{W\left(i \frac{\sqrt{2}}{\theta} \alpha\right) W\left(i \frac{\sqrt{2}}{\theta}(\alpha+\beta)\right)\right.
$$

$$
\left.+\frac{1}{2} \frac{\partial W}{\partial z}\left(z=i \frac{\sqrt{2}}{\theta} \alpha\right) \frac{\partial W}{\partial z}\left(z=i \frac{\sqrt{2}}{\theta}(\alpha+\beta)\right)\right\}
$$

To obtain the contribution of Eq. (5) for this case we once again set $\beta$ equal to zero, giving

$$
\begin{aligned}
\left\langle F_{6}^{4}\right\rangle= & \frac{(2 \pi)^{3 / 2}}{\left(2 \gamma_{e g}\right) \theta^{2}(k u)^{3}}\left\{\left[W\left(i \frac{\sqrt{2}}{\theta} \alpha\right)\right]^{2}\right. \\
& \left.+\frac{1}{2}\left[\frac{\partial W}{\partial z}\left(z=i \frac{\sqrt{2}}{\theta} \alpha\right)\right]^{2}\right\} .
\end{aligned}
$$

[1] Y. Prior, A. Bogdan, M. Dagenais, and N. Bloembergen, Phys. Rev. Lett. 46, 111 (1981).

[2] G. Grynberg and P. Berman, Phys. Rev. A 43, 3994 (1991).

[3] G. Agarwal and C. Kunasz, Phys. Rev. A 27, 996 (1983).

[4] Y. Prior, I. Scheck, and J. Jortner, Phys. Rev. A 31, 3775 (1985).

[5] A. Kofman, A. Levine, and Y. Prior, Phys. Rev. A 37, 1248 (1988).
[6] G. Vemuri, Phys. Rev. A 48, 3256 (1993).

[7] N. Morita and T. Yajima, Phys. Rev. A 30, 2525 (1984).

[8] G. Agarwal, C. Kunasz, and J. Cooper, Phys. Rev. A 36, 143 (1987).

[9] P. Tchenio, A. Debarre, J.-C. Keller, and J.-L. LeGouet, Phys. Rev. Lett. 62, 415 (1989).

[10] V. Finkelstein and P. Berman, Phys. Rev. A 41, 6193 (1990).

[11] V. Finkelstein and P. Berman, Phys. Rev. A 42, 3145 (1990). 
[12] R. Beach and S. Hartmann, Phys. Rev. Lett. 53, 663 (1984).

[13] M. Dugan and A. Albrecht, Phys. Rev. A 43, 3877 (1991).

[14] D. Ulness and A. Albrecht, Phys. Rev. A 53, 1081 (1996).

[15] R. Bratfalean and P. Ewart, Phys. Rev. A 56, 2267 (1997).

[16] S. Mukamel and E. Hanamura, Phys. Rev. A 33, 1099 (1986).

[17] R. Trebino, E. K. Gustafson, and A. E. Siegman, J. Opt. Soc.
Am. B 3, 1295 (1986).

[18] J. Goodman, Statistical Optics (Wiley-Interscience, New York, 1985).

[19] Handbook of Mathematical Functions, edited by M. Abramowitz and I. Stegun (Dover, New York, 1974). 